\title{
Generalized Riemann-Liouville Fractional Operators Associated with a Generalization of the Prabhakar Integral Operator
}

\author{
Gustavo A. Dorrego* \\ Department of Mathematics, Faculty of Exacts Sciences, Northeast National University, Av. Libertad 5460, Corrientes, Argentina
}

Received: 13 Sep. 2015, Revised: 18 Jan. 2016, Accepted: 22 Jan. 2016

Published online: 1 Apr. 2016

\begin{abstract}
The paper introduces a new integral operator which generalizes the Prabhakar integral operator. The boundedness on the space of continuous functions and on the space of Lebesgue integrable functions on an interval is studied. In addition, the left inverse operator is constructed. The properties of composition with the k-Riemann-Liouville fractional operators are analized. Finally, as an application, a fractional generalization of the Cauchy problem associated with free electron laser equation is proposed.
\end{abstract}

Keywords: Fractional integral operator, k-Gamma function, k-Mittag-Leffler function, Riemann-Lioville fractional integral, RiemannLiouville fractional derivative.

\section{Introduction}

In recent years, one focus of study in the field of fractional calculus was the generalization of integration and differentiation operators. In many generalizations of the integral operators appear special functions such as Gauss hypergeometric function, Mittag-Leffler type functions, Wright function, Meijer's G-function and Fox's H-function in the kernel of these operators. A very interesting work that meets many of these results is Operators of fractional integration and their applications by Srivastava and Saxena [1]. It is not mentioned in this work, the integral operator introduced by Prabhakar [2], which contains in its kernel a Mittag-Leffler type function of three parameters. In 2004, Kilbas et. al. [3] have studied this operator noting that generalizes the Riemann-Liouville fractional integral and proposed its left inverse operator, as a generalization of the Riemann-Liouville fractional derivative. In 2014, Garra et. al. [4] turned to the study of that operator using it to construct a fractional differential operator that generalizes the Hilfer fractional derivative.

Moreover, a crucial role in the field of fractional calculus is the Euler's Gamma function, which generalizes the factorial function and appears in the definitions of integral operators of non-integer order and at the definitions of special functions. Several generalizations of this function have been studied (see e.g. [5], [6]). One of these generalizations was introduced in 2007 by Diaz and Pariguan [7], namely the k-Gamma function. Since the k-Gamma function was introduced, many authors have presented extensions of some of the so-called special functions, such as the k-Mittag-Leffler function [8], k-Wright function [10], and k-Bessel functions [9,11]. In addition, also they have introduced generalizations of the classical fractional operators, (see [12], [13], [14], [15], [16], [17]). In this paper, we introduces a new generalization of the following integral operator due to Prabhakar:

$$
\left(\mathbf{E}_{\rho, \mu, \omega ; a^{+}}^{\gamma} \varphi\right)(x)=\int_{a}^{x}(x-t)^{\mu-1} E_{\rho, \mu}^{\gamma}\left[\omega(x-t)^{\rho}\right] \varphi(t) d t \quad(x>a)
$$

\footnotetext{
* Corresponding author e-mail: gusad82@gmail.com
} 
where $E_{\rho, \mu}^{\gamma}\left[\omega(x-t)^{\rho}\right]$, the Mittag-Leffler function defined in [2], will be replaced by the k-Mittag-Leffler function defined in [8], and given by

$$
E_{k, \alpha, \beta}^{\gamma}(z)=\sum_{n=0}^{\infty} \frac{(\gamma)_{n, k}}{\Gamma_{k}(\alpha n+\beta)} \frac{z^{n}}{n !}
$$

where $k \in \mathbb{R}^{+} ; \alpha, \beta, \gamma \in \mathbb{C} ; \operatorname{Re}(\alpha)>0, \operatorname{Re}(\beta)>0 ; \Gamma_{k}(x)$ it is the k-Gamma function given by $(4)$ and $(\gamma)_{n, k}=\frac{\Gamma_{k}(\gamma+n k)}{\Gamma_{k}(\gamma)}$ it is the Pochhammer k-symbol.

In the following, we will highlight some points needed for the sequel.

In 2012 Mubeen and Habbibulah [15] introduced the k-Riemann-Liouville fractional integral given by

Definition 1. Let $\alpha \in \mathbb{R}^{+}$and $n \in \mathbb{N}$ such that $n-1<\alpha<n, f \in L^{1}([0, \infty))$. Then the $k$-Riemann-Liouville fractional integral of $f$ is

$$
I_{k}^{\alpha} f(t)=\frac{1}{k \Gamma_{k}(\alpha)} \int_{0}^{t}(t-\tau)^{\frac{\alpha}{k}-1} f(\tau) d \tau=\frac{t^{\frac{\alpha}{k}-1}}{k \Gamma_{k}(\alpha)} * f(t), t>0,
$$

where

$$
\Gamma_{k}(\alpha)=\int_{0}^{\infty} t^{\alpha-1} e^{-\frac{t^{k}}{k}} d t, k>0
$$

is the k-Gamma function introduced in [7] and whose relationship with the classical Gamma function is

$$
\Gamma_{k}(\alpha)=k^{\frac{\alpha}{k}-1} \Gamma\left(\frac{\alpha}{k}\right)
$$

Since the k-Gamma function is such that $\Gamma_{k}(\alpha) \rightarrow \Gamma(\alpha)$ when $k \rightarrow 1$, it follows that $I_{k}^{\alpha} \rightarrow I^{\alpha}$.

The k-integral (3) also satisfies the semigroup property

Proposition 1. Let $\alpha, \beta \in \mathbb{R}^{+}, f \in L^{1}([0, \infty))$ and $k>0$, then

For the proof, we remit to [15] formula (10) p. 91.

$$
I_{k}^{\alpha} I_{k}^{\beta} f(t)=I_{k}^{\alpha+\beta} f(t)=I_{k}^{\beta} I_{k}^{\alpha} f(t) .
$$

The left inverse operator of (3) was defined and studied by the author in [13], and it is given by the following

Definition 2. Let $k, \alpha \in \mathbb{R}^{+}$and $n \in \mathbb{N}$ such that $n=\left[\frac{\alpha}{k}\right]+1, f \in L^{1}([0, \infty))$ and $I_{k}^{n k-\alpha} f(t) \in W^{n, 1}[0, \infty)$; the k-RiemannLiouville fractional derivative is given by

$$
\mathfrak{k}_{R L}^{\alpha} f(t)=\left(\frac{d}{d t}\right)^{n} k^{n} I_{k}^{n k-\alpha} f(t)
$$

where $W^{n, 1}[a, b]$ denotes the Sobolev space $W^{n, 1}[a, b]=\left\{f \in L^{1}[a, b]: f^{(n)} \in L^{1}[a, b]\right\}$.

Remark. If $k=1$ (7) coincides with the classical Riemann-Liouville fractional derivative.

Remark. In Definitions 1.1 and 1.2 is to possible consider $\alpha \in \mathbb{C}(\Re(\alpha)>0)$. Therefore, in the Definition 1.2 must be $n=\left[\frac{\Re(\alpha)}{k}\right]+1$

\section{Results}

Definition 3.(k-Prabhakar integral) Let $\alpha, \beta, \omega, \gamma, \in \mathbb{C}, k \in \mathbb{R}^{+} ; \mathfrak{R}(\alpha)>0 ; \mathfrak{R}(\beta)>0$ and $\varphi \in L^{1}([0, b]),(0<x<$ $b \leq \infty)$. The $k$-Prabhakar integral operator is defined as

$$
\begin{aligned}
\left({ }_{k} \mathbf{P}_{\alpha, \beta, \omega}^{\gamma} \varphi\right)(x) & =\int_{0}^{x} \frac{(x-t)^{\frac{\beta}{k}-1}}{k} E_{k, \alpha, \beta}^{\gamma}\left[\omega(x-t)^{\frac{\alpha}{k}}\right] \varphi(t) d t, \quad(x>0) \\
& =\left({ }_{k} \mathscr{E}_{\alpha, \beta, \omega}^{\gamma} * f\right)(x),
\end{aligned}
$$

where

$$
{ }_{k} \mathscr{E}_{\alpha, \beta, \omega}^{\gamma}(t)= \begin{cases}\frac{t^{\frac{\beta}{k}-1}}{k} E_{k, \alpha, \beta}^{\gamma}\left(\omega t^{\frac{\alpha}{k}}\right), & t>0 \\ 0, & t \leq 0\end{cases}
$$


Remark. Note here that for $\gamma=0$ we have

$$
\left({ }_{k} \mathbf{P}_{\alpha, \beta, \omega}^{0} \varphi\right)(t)=\left(I_{k}^{\beta} \varphi\right)(t)
$$

i.e. the operator (8) generalizes the k-Riemann-Liouville fractional integral defined by (3).

It is interesting to study the boundedness of the k-Prabhakar integral on different spaces, namely, on the space of Lebesgue integrable functions and on the space of continuous functions on a closed interval. Indeed, we have the following two Propositions.

Proposition 2. The $k$-Prabhakar integral is bounded on $L^{1}([0, b]),(0<x \leq b<\infty)$.

Let $\alpha, \beta, \omega, \gamma, \in \mathbb{C}, k \in \mathbb{R}^{+} ; \Re(\alpha)>0 ; \Re(\beta)>0$ and $\varphi \in L^{1}([0, b])$ hence

$$
\left\|\left({ }_{k} \mathbf{P}_{\alpha, \beta, \omega}^{\gamma} \varphi\right)(x)\right\|_{1} \leq B\|\varphi\|_{1}
$$

where

$$
B=\frac{b^{\Re\left(\frac{\beta}{k}\right)}}{k} \sum_{n=0}^{\infty} \frac{\left|(\gamma)_{n, k}\left(\omega b^{\Re\left(\frac{\alpha}{k}\right)}\right)^{n}\right|}{\left[n \Re\left(\frac{\alpha}{k}\right)+\Re\left(\frac{\beta}{k}\right)\right]\left|\Gamma_{k}(\alpha n+\beta)\right| n !}
$$

Proof.

First, we will prove that the series in (13) it is convergent.

Denoting by $c_{n}$ the nth term of the series, and using (5) and the following relations

$$
(\gamma)_{n, k}=k^{n}\left(\frac{\gamma}{k}\right)_{n}
$$

and

$$
\frac{\Gamma(z+\rho)}{\Gamma(z+\mu)}=z^{\rho-\mu}\left[1+\frac{1}{2 z}(\rho-\mu)(\rho+\mu-1)+O\left(z^{-2}\right)\right]
$$

for $|z| \rightarrow \infty,|\arg (z)| \leq \pi-\varepsilon, \mid \arg (z+\rho \mid \leq \pi-\varepsilon, 0<\varepsilon<\pi$;

we finally obtain

$$
\begin{gathered}
\left|\frac{c_{n+1}}{c_{n}}\right|=k^{-\frac{\alpha}{k}} \frac{|n+\gamma / k|}{n+1}\left|\frac{\Gamma\left(\frac{\alpha}{k} n+\frac{\beta}{k}\right)}{\Gamma\left(\frac{\alpha}{k} n+\frac{\beta}{k}+\frac{\alpha}{k}\right)}\right| \\
\times \frac{\left[n \Re\left(\frac{\alpha}{k}\right)+\Re\left(\frac{\beta}{k}\right)\right]}{\left[(n+1) \Re\left(\frac{\alpha}{k}\right)+\Re\left(\frac{\beta}{k}\right)\right]}|\omega| b^{\Re\left(\frac{\alpha}{k}\right)} \sim \frac{|\omega| b^{\Re\left(\frac{\alpha}{k}\right)}}{\left(\left|\frac{\alpha}{k}\right| n\right)^{\Re\left(\frac{\alpha}{k}\right)} \rightarrow 0(n \rightarrow \infty)}
\end{gathered}
$$

which means that the right-hand side of (13) is convergent and thus B is finite.

Now, we will prove (12). We consider $\varphi \in L^{1}([0, b])$ then by using (8), interchanging the order of integration and taking $\tau=x-t$ result

$$
\begin{aligned}
& \left\|\left({ }_{k} \mathbf{P}_{\alpha, \beta, \omega}^{\gamma} \varphi\right)(x)\right\|_{1}=\int_{0}^{b} \frac{1}{k}\left|\int_{0}^{x}(x-t)^{\frac{\beta}{k}-1} E_{k, \alpha, \beta}^{\gamma}\left[\omega(x-t)^{\frac{\alpha}{k}}\right] \varphi(t) d t\right| d x \\
& \leq \frac{1}{k} \int_{0}^{b}\left[\int_{t}^{b}(x-t)^{\Re\left(\frac{\beta}{k}\right)-1}\left|E_{k, \alpha, \beta}^{\gamma}\left[\omega(x-t)^{\frac{\alpha}{k}}\right]\right| d x\right]|\varphi(t)| d t \\
& =\frac{1}{k} \int_{0}^{b}\left[\int_{0}^{b-t} \tau^{\Re\left(\frac{\beta}{k}\right)-1}\left|E_{k, \alpha, \beta}^{\gamma}\left[\omega \tau^{\frac{\alpha}{k}}\right]\right| d \tau\right]|\varphi(t)| d t \\
& \leq \int_{0}^{b}[\underbrace{\frac{1}{k} \int_{0}^{b} \tau^{\Re\left(\frac{\beta}{k}\right)-1}\left|E_{k, \alpha, \beta}^{\gamma}\left[\omega \tau^{\frac{\alpha}{k}}\right]\right| d \tau}_{\Omega}]|\varphi(t)| d t .
\end{aligned}
$$


We note that

$$
\Omega \leq \frac{1}{k} \sum_{n=0}^{\infty} \frac{\left|(\gamma)_{n, k}\right||\omega|^{n}}{\left|\Gamma_{k}(\alpha n+\beta)\right| n !} \int_{0}^{b} \tau^{\Re\left(\frac{\alpha}{k}\right) n+\Re\left(\frac{\beta}{k}\right)-1} d \tau=B
$$

Then, from (16) and (17) we have (12).

Proposition 3. The k-Prabhakar integral is bounded on $C([0, x]),(0<x \leq b<\infty)$. Let $\alpha, \beta, \omega, \gamma, \in \mathbb{C}, k \in \mathbb{R}^{+} ; \Re(\alpha)>$ $0 ; \mathfrak{R}(\beta)>0$ and $\varphi \in C([0, b])$ hence

$$
\left\|\left({ }_{k} \mathbf{P}_{\alpha, \beta, \omega}^{\gamma} \varphi\right)(x)\right\|_{C} \leq B\|\varphi\|_{C}
$$

where

$$
\|\varphi\|_{C}=\max \{|\varphi|: 0 \leq x \leq b\}
$$

and $B$ is given by (13).

Proof.

Let us $\varphi \in C([0, b])$ and $x \in[0, b]$, and taking into account (19) we have

$$
\begin{aligned}
\left|\left({ }_{k} \mathbf{P}_{\alpha, \beta, \omega}^{\gamma} \varphi\right)(x)\right| & \leq \int_{0}^{x}\left|(x-t)^{\frac{\beta}{k}-1} E_{k, \alpha, \beta}^{\gamma}\left[\omega(x-t)^{\frac{\alpha}{k}}\right]\right||\varphi(t)| d t \\
& \leq\|\varphi(t)\|_{C} \int_{0}^{x}(x-t)^{\Re\left(\frac{\beta}{k}\right)-1}\left|E_{k, \alpha, \beta}^{\gamma}\left[\omega(x-t)^{\frac{\alpha}{k}}\right]\right| d t .
\end{aligned}
$$

Repeating what was done in (16) and (17) and considering that $0 \leq x \leq b$, the integral in (20) is less than or equal to $B$. This completes the proof of (18).

We now calculate the operator applied to certain functions such as potential function and k-Mittag-Leffler function. To this end, first we demonstrate the following lemmas.

Lemma 1. Let $\alpha, \beta, \omega, \gamma, \in \mathbb{C}, k \in \mathbb{R}^{+} ; \mathfrak{R}(\alpha)>0 ; \mathfrak{R}(\beta)>0$. Then

$$
I_{k}^{\alpha}\left[(t-\tau)^{\frac{\beta}{k}-1} E_{k, \rho, \beta}^{\gamma}\left(\omega(t-\tau)^{\frac{\rho}{k}}\right)\right]=(t-\tau)^{\frac{\alpha+\beta}{k}-1} E_{k, \rho, \beta+\alpha}^{\gamma}\left(\omega(t-\tau)^{\frac{\rho}{k}}\right)
$$

Proof. Starting on the left-hand side, using (3) and (8), the uniform convergence of the serie (2) and taking into account ([15], f. 12) we have (21).

$$
\begin{aligned}
I_{k}^{\alpha}\left[(t-\tau)^{\frac{\beta}{k}-1} E_{k, \rho, \beta}^{\gamma}\left(\omega(t-\tau)^{\frac{\rho}{k}}\right)\right] & =\frac{1}{k \Gamma_{k}(\alpha)} \int_{0}^{t}(t-\tau)^{\frac{\alpha}{k}-1}(t-\tau)^{\frac{\beta}{k}-1} E_{k, \rho, \beta}^{\gamma}\left(\omega(t-\tau)^{\frac{\rho}{k}}\right) d \tau \\
& =\sum_{n=0}^{\infty} \frac{(\gamma)_{n, k} \omega^{n}}{k \Gamma_{k}(\rho n+\beta) n !} \frac{1}{\Gamma_{k}(\alpha)} \int_{0}^{t}(t-\tau)^{\frac{\rho n+\beta}{k}}-1(t-\tau)^{\frac{\alpha}{k}} d \tau \\
& =\sum_{n=0}^{\infty} \frac{(\gamma)_{n, k} \omega^{n}}{n !} \frac{1}{\Gamma_{k}(\alpha)} I_{k}^{\rho n+\beta}\left[(t-\tau)^{\frac{\alpha}{k}}\right] \\
& =(t-\tau)^{\frac{\beta+\alpha}{k}-1} E_{k, \rho, \beta+\alpha}^{\gamma}\left(\omega(t-\tau)^{\frac{\rho}{k}}\right) .
\end{aligned}
$$

Lemma 2. Let $\alpha, \beta, \omega, \gamma, \in \mathbb{C}, k \in \mathbb{R}^{+} ; \mathfrak{R}(\alpha)>0 ; \Re(\beta)>0$ and $\varphi \in L^{1}\left(\mathbb{R}_{0}^{+}\right)$and $\left|\omega k(k s)^{-\frac{\rho}{k}}\right|<1$. Then

$$
\begin{aligned}
\mathscr{L}\left\{\left({ }_{k} \mathbf{P}_{\rho, \beta, \omega}^{\gamma} \varphi\right)(x)\right\}(s) & =\mathscr{L}\left\{{ }_{k} \mathscr{E}_{\rho, \beta, \omega}^{\gamma}(t)\right\}(s) \mathscr{L}\{\varphi\}(s) \\
& =(k s)^{-\frac{\beta}{k}}\left(1-\omega k(k s)^{-\frac{\rho}{k}}\right)^{-\frac{\gamma}{k}} \mathscr{L}\{\varphi\}(s) .
\end{aligned}
$$

Proof. It is sufficient to calculate the Laplace transform of the kernel (10). For this purpose, taking into account (5) and the generalized binomial theorem, we have

$$
\sum_{n=0}^{\infty} \frac{(\gamma)_{n, k} \omega^{n}}{n !}=(1-k \omega)^{-\frac{\gamma}{k}}, \quad|k \omega|<1
$$




$$
\begin{aligned}
\mathscr{L}\left\{{ }_{k} \mathscr{E}_{\alpha, \beta, \omega}^{\gamma}(t)\right\}(s) & =\frac{1}{k} \int_{0}^{\infty} e^{-s t} t^{\frac{\beta}{k}-1} E_{k, \alpha, \beta}^{\gamma}\left(\omega t^{\frac{\alpha}{k}}\right) d t \\
& =\frac{1}{k} \sum_{n=0}^{\infty} \frac{(\gamma)_{n, k} \omega^{n}}{\Gamma_{k}(\alpha n+\beta) n !} \int_{0}^{\infty} e^{-s t} t^{\frac{\alpha}{k} n+\frac{\beta}{k}-1} d t \\
& =\frac{1}{k} \sum_{n=0}^{\infty} \frac{(\gamma)_{n, k} \omega^{n}}{\Gamma_{k}(\alpha n+\beta) n !} \frac{\Gamma\left(\frac{\alpha n+\beta}{k}\right)}{s^{\frac{\alpha n+\beta}{k}}} \\
& =\frac{1}{k} \sum_{n=0}^{\infty} \frac{(\gamma)_{n, k} \omega^{n}}{n ! k^{\frac{\alpha n+\beta}{k}-1} s^{\frac{\alpha n+\beta}{k}}} \\
& =\frac{1}{(k s)^{\frac{\beta}{k}}} \sum_{n=0}^{\infty} \frac{(\gamma)_{n, k}}{n !}\left(\frac{\omega}{(k s)^{\frac{\alpha}{k}}}\right)^{n} \\
& =(k s)^{-\frac{\beta}{k}}\left(1-\omega k(k s)^{-\frac{\alpha}{k}}\right)^{-\frac{\gamma}{k}}, \quad\left|\frac{k \omega}{(k s)^{\frac{\alpha}{k}}}\right|<1 .
\end{aligned}
$$

Proposition 4. Let $\rho, \beta, \omega, \gamma \in \mathbb{C}, k \in \mathbb{R}^{+}$and $\Re(\rho)>0, \Re(\beta)>0, \Re(\alpha)>0$. Then

$$
\left({ }_{k} \mathbf{P}_{\rho, \beta, \omega}^{\gamma}\right)\left[\sigma^{\frac{\alpha}{k}-1}\right](t)=\Gamma_{k}(\alpha) t^{\frac{\alpha+\beta}{k}-1} E_{k, \rho, \beta+\alpha}^{\gamma}\left(\omega t^{\frac{\rho}{k}}\right) .
$$

Proof.

Taking into account ([15], f.11) we have

$$
\begin{aligned}
\left({ }_{k} \mathbf{P}_{k, \rho, \beta, \omega}^{\gamma}\right)\left[\sigma^{\frac{\alpha}{k}-1}\right](t) & =\int_{0}^{t} \frac{(t-\sigma)^{\frac{\beta}{k}-1}}{k} E_{k, \rho, \beta}^{\gamma}\left(\omega(t-\sigma)^{\frac{\rho}{k}}\right) \sigma^{\frac{\alpha}{k}-1} d \sigma \\
& =\int_{0}^{t} \frac{1}{k} \sum_{n=0}^{\infty} \frac{(\gamma)_{n, k} \omega^{n}(t-\sigma)^{\frac{\rho n+\beta}{k}}-1 \sigma^{\frac{\alpha}{k}-1}}{\Gamma_{k}(\rho n+\beta) n !} d \sigma \\
& =\sum_{n=0}^{\infty} \frac{(\gamma)_{n, k} \omega^{n}}{n !} \frac{1}{k \Gamma_{k}(\rho n+\beta)} \int_{0}^{t}(t-\sigma)^{\frac{\rho n+\beta}{k}-1} \sigma^{\frac{\alpha}{k}-1} d \sigma \\
& =\sum_{n=0}^{\infty} \frac{(\gamma)_{n, k} \omega^{n}}{n !} I_{k}^{\rho n+\beta}\left[\sigma^{\frac{\alpha}{k}-1}\right](t) \\
& =\sum_{n=0}^{\infty} \frac{(\gamma)_{n, k} \omega^{n}}{n !} \frac{\Gamma_{k}(\alpha)}{\Gamma_{k}(\rho n+\beta+\alpha)} t^{\frac{\rho n+\beta+\alpha}{k}-1} \\
& =\Gamma_{k}(\alpha) t^{\frac{\alpha+\beta}{k}-1} E_{k, \rho, \alpha+\beta}^{\gamma}\left(\omega t^{\frac{\rho}{k}}\right) .
\end{aligned}
$$

Proposition 5. Let $\rho, \beta, \mu, \delta, \omega, \gamma \in \mathbb{C}, k \in \mathbb{R}^{+} ; \Re(\rho)>0, \Re(\beta)>0$ and $\left|\frac{k \omega}{(k s)^{\frac{\alpha}{k}}}\right|<1$. Then

$$
\left({ }_{k} \mathbf{P}_{\rho, \beta, \omega}^{\gamma}\right)\left[\frac{\sigma^{\frac{\mu}{k}-1}}{k} E_{k, \rho, \mu}^{\delta}\left(\omega(x-\sigma)^{\frac{\rho}{k}}\right)\right](t)=\frac{t^{\frac{\mu+\beta}{k}}-1}{k} E_{k, \rho, \mu+\beta}^{\delta+\gamma}\left(\omega(x-t)^{\frac{\rho}{k}}\right) .
$$

Proof.

The left hand side of (32), according to (8) can be written as

$$
\left({ }_{k} \mathscr{E}_{\rho, \beta, \omega}^{\gamma} *{ }_{k} \mathscr{E}_{\rho, \mu, \omega}^{\delta}\right)(t)
$$

Now, taking Laplace transform and applying the convolution theorem for the Laplace transform and Lemma 2

$$
\begin{aligned}
\mathscr{L}\left\{\left({ }_{k} \mathscr{E}_{\rho, \beta, \omega}^{\gamma} *{ }_{k} \mathscr{E}_{\rho, \mu, \omega}^{\delta}\right)(t)\right\}(s) & =\mathscr{L}\left\{{ }_{k} \mathscr{E}_{\rho, \beta, \omega}^{\gamma}(t)\right\}(s) \mathscr{L}\left\{{ }_{k} \mathscr{E}_{\rho, \mu, \omega}^{\delta}(t)\right\}(s) \\
& =(k s)^{-\frac{\beta+\mu}{k}}\left(1-\omega k(k s)^{-\frac{\rho}{k}}\right)^{-\frac{\gamma+\delta}{k}}
\end{aligned}
$$

Then because the inverse Laplace transform, the results can be achieved. 


\subsection{Compositions of k-Fractional Calculus Operators with the k-Prabhakar Integral Operator}

We now consider composition with the k-Riemann-Liouville fractional integral.

Proposition 6. Let $\alpha \in \mathbb{C},(\Re(\alpha))$, and $\rho, \beta, \omega, \gamma \in \mathbb{C},(\Re(\rho)>0, \Re(\beta)>0)$. Then relation for any function $f \in L^{1}([0, b]),(0<x<b \leq \infty)$ hold

$$
I_{k}^{\alpha}\left({ }_{k} \mathbf{P}_{\rho, \beta, \omega}^{\gamma} f(t)\right)={ }_{k} \mathbf{P}_{\rho, \beta+\alpha, \omega}^{\gamma} f(t)={ }_{k} \mathbf{P}_{\rho, \beta, \omega}^{\gamma}\left(I_{k}^{\alpha} f(t)\right)
$$

Proof.

We will start proving the first equality in (34),

$$
\begin{gathered}
I_{k}^{\alpha}\left({ }_{k} \mathbf{P}_{\rho, \beta, \omega}^{\gamma} f(t)\right)=\frac{1}{k \Gamma_{k}(\alpha)} \int_{0}^{t}(t-x)^{\frac{\alpha}{k}-1}{ }_{k} \mathbf{P}_{\rho, \beta, \omega}^{\gamma} f(x) d x \\
=\frac{1}{k \Gamma_{k}(\alpha)} \int_{0}^{t}(t-x)^{\frac{\alpha}{k}-1} \int_{0}^{x} \frac{(x-\tau)^{\frac{\beta}{k}-1}}{k} E_{k, \rho, \beta}^{\gamma}\left[\omega(x-\tau)^{\frac{\rho}{k}}\right] f(\tau) d \tau d x .
\end{gathered}
$$

By inverting the order of integration,

$$
\begin{aligned}
& \frac{1}{k^{2} \Gamma_{k}(\alpha)} \int_{0}^{t} \int_{\tau}^{t}(x-\tau)^{\frac{\beta}{k}-1}(t-x)^{\frac{\alpha}{k}-1} E_{k, \rho, \beta}^{\gamma}\left[\omega(x-\tau)^{\frac{\rho}{k}}\right] d x d \tau \\
= & \frac{1}{k^{2} \Gamma_{k}(\alpha)} \int_{0}^{t} f(\tau) \int_{\tau}^{t}(x-\tau)^{\frac{\beta}{k}-1}(t-x)^{\frac{\alpha}{k}-1} E_{k, \rho, \beta}^{\gamma}\left[\omega(x-\tau)^{\frac{\rho}{k}}\right] d x d \tau
\end{aligned}
$$

making the change of variable $x-\tau=\xi$ we have

$$
\begin{gathered}
\frac{1}{k^{2} \Gamma_{k}(\alpha)} \int_{0}^{t} f(\tau) \int_{0}^{t-\tau}(t-\tau-\xi)^{\frac{\alpha}{k}-1} \xi^{\frac{\beta}{k}-1} E_{k, \rho, \beta}^{\gamma}\left[\omega \xi^{\frac{\rho}{k}}\right] d \xi d \tau \\
=\frac{1}{k} \int_{0}^{t} I_{k}^{\alpha}\left[(t-\tau)^{\frac{\beta}{k}-1} E_{k, \rho, \beta}^{\gamma}\left[\omega(t-\tau)^{\frac{\rho}{k}}\right]\right] f(\tau) d \tau
\end{gathered}
$$

and by (21) finally obtains

$$
\frac{1}{k} \int_{0}^{t}(t-\tau)^{\frac{\beta+\alpha}{k}-1} E_{k, \rho, \beta+\alpha}^{\gamma}\left[\omega(t-\tau)^{\frac{\rho}{k}}\right] f(\tau) d \tau={ }_{k} \mathbf{P}_{\rho, \beta+\alpha, \omega} f(t) .
$$

To prove the second equality, with an analogous procedure, taking $\sigma=\tau-\xi$, we have

${ }_{k} \mathbf{P}_{\rho, \beta, \omega}^{\gamma}\left(I_{k}^{\alpha} f(t)\right)=\frac{1}{k \Gamma_{k}(\alpha)} \int_{0}^{t}\left({ }_{k} \mathbf{P}_{\rho, \beta, \omega}\left(\sigma^{\frac{\alpha}{k}-1}\right)\right)(t-\xi) f(\xi) d \xi$

Taking into account (31) we get

$$
\begin{gathered}
\frac{1}{k \Gamma_{k}(\alpha)} \int_{0}^{t}\left({ }_{k} \mathbf{P}_{\rho, \beta, \omega}\left(\sigma^{\frac{\alpha}{k}-1}\right)\right)(t-\xi) f(\xi) d \xi=\frac{\Gamma_{k}(\alpha)}{k \Gamma_{k}(\alpha)} \int_{0}^{t}(t-\xi)^{\frac{\beta+\alpha}{k}-1} E_{k, \rho, \beta+\alpha}^{\gamma}\left[\omega(t-\xi)^{\frac{\rho}{k}}\right] f(\xi) d \xi \\
={ }_{k} \mathbf{P}_{\rho, \beta+\alpha}^{\gamma} f(t) .
\end{gathered}
$$

Then, from (37) and (38), (34) is obtained.

Now we study the composition with the k-fractional differential operator $\mathfrak{D}_{R L}^{\alpha}$.

Proposition 7. Let $\alpha, \rho, \beta, \gamma, \omega \in \mathbb{C} ; k \in \mathbb{R}^{+}, \mathfrak{R}(\alpha)>0, \Re(\rho)>0, \Re(\beta)>0$; then for $f \in L^{1}([0, b])$ and $0<x<b \leq \infty$ hold

$$
{ }_{k} \mathfrak{D}_{R L}^{\alpha}\left({ }_{k} \mathbf{P}_{\rho, \beta, \omega}^{\gamma} f(t)\right)={ }_{k} \mathbf{P}_{\rho, \beta-\alpha, \omega}^{\gamma} f(t) .
$$


Proof.

Let us suppose $n=\left[\frac{\alpha}{k}\right]+1$, then by (7) and (34) we have:

$$
\begin{gathered}
{ }_{k} \mathfrak{D}_{R L}^{\alpha}\left({ }_{k} \mathbf{P}_{\rho, \beta, \omega}^{\gamma} f(t)\right)=\left(\frac{d}{d t}\right)^{n} k^{n} I_{k}^{n k-\alpha}{ }_{k} \mathbf{P}_{\rho, \beta, \omega}^{\gamma} f(t) \\
=k^{n}\left(\frac{d}{d t}\right)^{n}{ }_{k} \mathbf{P}_{\rho, \beta+n k-\alpha, \omega}^{\gamma} f(t) \\
=k^{n-1}\left(\frac{d}{d t}\right)^{n} \int_{0}^{t}(t-x)^{\frac{\beta+n k-\alpha}{k}-1} E_{k, \rho, \beta+n k-\alpha}^{\gamma}\left(\omega(t-x)^{\frac{\alpha}{k}}\right) f(x) d x \\
=\frac{1}{k} \int_{0}^{t}(t-x)^{\frac{\beta-\alpha}{k}-1} E_{k, \rho, \beta-\alpha}^{\gamma}\left(\omega(t-x)^{\frac{\alpha}{k}}\right) f(x) d x={ }_{k} \mathbf{P}_{\rho, \beta-\alpha, \omega}^{\gamma} f(t) .
\end{gathered}
$$

Another important property of the operator (8) is the semigroup property.

Proposition 8. Let $k \in \mathbb{R}^{+}$and $\rho, \beta, \gamma, v, \delta, \omega \in \mathbb{C}, \mathfrak{R}(\rho)>0, \Re(\beta)>0, \Re(v)>0$; then for any $\varphi \in L^{1}([0, b])$ and $0<x<b \leq \infty$ we have

$$
\left.{ }_{k} \mathbf{P}_{\rho, \beta, \omega}^{\gamma}\left({ }_{k} \mathbf{P}_{\rho, v, \omega}^{\delta} \varphi\right)(t)=\left({ }_{k} \mathbf{P}_{\rho, \beta+v, \omega}^{\gamma+\delta} \varphi\right)(t)={ }_{k} \mathbf{P}_{\rho, v, \omega}^{\delta}{ }_{k} \mathbf{P}_{\rho, \beta, \omega}^{\gamma} \varphi\right)(t)
$$

As particular case

$$
{ }_{k} \mathbf{P}_{\rho, \beta, \omega}^{\gamma}\left({ }_{k} \mathbf{P}_{\rho, v, \omega}^{-\gamma} \varphi\right)(t)=I_{k}^{\beta+v} \varphi(t)
$$

Proof.

Interchanging the order of integration, taking $\tau=\mu-x$, and finally the formula (32) is

$$
\begin{aligned}
{ }_{k} \mathbf{P}_{\rho, \beta, \omega}^{\gamma}\left({ }_{k} \mathbf{P}_{\rho, v, \omega}^{\delta} \varphi\right)(t) & =\frac{1}{k} \int_{0}^{t}(t-x)^{\frac{\beta+v}{k}-1} E_{k, \rho, \beta+v}^{\gamma+\delta}\left(\omega(t-x)^{\frac{\rho}{k}}\right) \varphi(x) d x \\
& =\left({ }_{k} \mathbf{P}_{\rho, \beta+v, \omega}^{\gamma+\delta} \varphi\right)(t) .
\end{aligned}
$$

\subsection{The Inverse Operator}

We here construct the left inverse operator. To do that, we propose the following Volterra integral equation of the first kind.

$$
\left({ }_{k} \mathbf{P}_{\rho, \beta, \omega}^{\gamma} \varphi\right)(x)=f(x), \quad \varphi(x) \in L^{1}([0, \infty)) .
$$

Taking into account (12) we have that $f \in L^{1}([0, \infty))$, then given $v \in \mathbb{C}, \Re(v)>0$, by composition with the operator ${ }_{k} \mathbf{P}_{\rho, v, \omega}^{-\gamma}$ and by the property (42) we have,

$$
\begin{aligned}
{ }_{k} \mathbf{P}_{\rho, v, \omega}^{-\gamma}\left({ }_{k} \mathbf{P}_{\rho, \beta, \omega}^{\gamma} \varphi\right)(x) & ={ }_{k} \mathbf{P}_{\rho, v, \omega}^{-\gamma} f(x) \\
I_{k}^{\beta+v} \varphi(x) & ={ }_{k} \mathbf{P}_{\rho, v, \omega}^{-\gamma} f(x) .
\end{aligned}
$$

Since $\Re(\beta+v)>0$ and $f \in L^{1}([0, \infty))$, then by Definition 6 of [13], we can apply the k-Riemann-Liouville fractional derivative (7) of order $\beta+v$, and we obtain

$$
\varphi(x)={ }_{k} \mathfrak{D}_{R L}^{\beta+v}{ }_{k} \mathbf{P}_{\rho, v, \omega}^{-\gamma} f(x),
$$

which is the solution of (43).

In conclusion, the left inverse operator of ${ }_{k} \mathbf{P}_{\rho, \beta, \omega}^{\gamma}$ is ${ }_{k} \mathfrak{D}_{R L}^{\beta+v}{ }_{k} \mathbf{P}_{\rho, v, \omega}^{-\gamma}$, i.e.

$$
\left[{ }_{k} \mathbf{P}_{\rho, \beta, \omega}^{\gamma}\right]^{-1}={ }_{k} \mathfrak{D}_{R L}^{\beta+v}{ }_{k} \mathbf{P}_{\rho, v, \omega}^{-\gamma} .
$$


Remark. Note here that putting $k=1$, we have that (47) coincides with the inversion formula given in Theorem 9 of [3].

To obtain an equivalent expression to (47), we introduce the following

Definition 4.(The k-Prabhakar derivative) $\quad$ Let $k \in \mathbb{R}^{+}, \rho, \beta, \gamma, \omega \in \mathbb{C}, \mathfrak{R}(\rho)>0, \mathfrak{R}(\beta)>0, m=\left[\frac{\beta}{k}\right]+1$ and $f \in$ $L^{1}([0, b])$. We define the $k$-Prabhakar derivative by

$$
{ }_{k} \mathbf{D}_{\rho, \beta, \omega}^{\gamma} f(x)=\left(\frac{d}{d x}\right)^{m} k_{k}^{m} \mathbf{P}_{\rho, m k-\beta, \omega}^{-\gamma} f(x) .
$$

We note that (47) and (48) are equivalent. Indeed, proceeding analogously to what was done in [4], taking $v \in \mathbb{C}, \mathfrak{R}(v)>0, p=\left[\frac{\Re(\beta)+\Re(v)}{k}\right]+1$, by (II.9) of [13] we have

$$
\begin{aligned}
{ }_{k} \mathbf{D}_{\rho, \beta, \omega}^{\gamma} f(x) & =\left(\frac{d}{d x}\right)^{m} k_{k}^{m} \mathbf{P}_{\rho, m k-\beta, \omega}^{-\gamma} f(x) \\
& =\left(\frac{d}{d x}\right)^{m} k^{m}\left(\frac{d}{d x}\right)^{p-m} k^{p-m} I_{k}^{(p-m) k}{ }_{k} \mathbf{P}_{\rho, m k-\beta, \omega}^{-\gamma} f(x) \\
& =\left(\frac{d}{d x}\right)^{p} k^{p}{ }_{k} \mathbf{P}_{\rho, m k-\beta+p k-m k, \omega}^{-\gamma} f(x) \\
& =\left(\frac{d}{d x}\right)^{p} k^{p}{ }_{k} \mathbf{P}_{\rho, p k-\beta, \omega}^{-\gamma} f(x) \\
& =\left(\frac{d}{d x}\right)^{p} k_{k}^{p}{ }_{k} \mathbf{P}_{\rho, p k-(\beta+v), \omega}^{0} \mathbf{P}_{\rho, v, \omega}^{-\gamma} f(x), v \in \mathbb{C}, \mathfrak{R}(v)>0 \\
& =\left(\frac{d}{d x}\right)^{p} k^{p} I_{k}^{p k-(\beta+v)}{ }_{k} \mathbf{P}_{\rho, v, \omega}^{-\gamma} f(x) \\
& ={ }_{k} \mathfrak{D}_{R L}^{\beta+v}{ }_{k} \mathbf{P}_{\rho, v, \omega}^{-\gamma} f(x) .
\end{aligned}
$$

Therefore, (48) is the left inverse operator of (8).

Remark. If $\gamma=0$ in (48) then the k-Prabhakar derivative coincides with the k-Riemann-Liouville derivative given in [13]. Indeed,

$$
\begin{aligned}
{ }_{k} \mathbf{D}_{\rho, \beta, \omega}^{0} f(x) & =\left(\frac{d}{d x}\right)^{m} k^{m}{ }_{k} \mathbf{P}_{\rho, m k-\beta, \omega}^{0} f(x) \\
& =\left(\frac{d}{d x}\right)^{m} k^{m} I_{k}^{m k-\beta} f(x) \\
& ={ }_{k} \mathfrak{D}_{R L}^{\beta} f(x) .
\end{aligned}
$$

\section{A Generalization of the Free Electron Laser Equation}

In this section we consider an equation that generalizes formula (1.1) of [14], in the case a $=0$, and that contains, as a particular case, the free electron laser equation.

Theorem 1. Given the following Cauchy problem

$$
\begin{cases}{ }_{k} \mathbf{D}_{\rho, \beta, \omega}^{\gamma} y(x)=\lambda_{k} \mathbf{P}_{\rho, v, \omega}^{\delta} y(x)+f(x), & f \in L^{1}[0, \infty) \\ \left({ }_{k} \mathbf{P}_{\rho, k-\beta, \omega}^{-\gamma} y\right)(0)=c, & c \geq 0\end{cases}
$$

where $\left[\frac{\beta}{k}\right]+1=m=1, \omega, \lambda \in \mathbb{C}, \rho>0, v>0, \gamma \geq 0, \delta \geq 0$, we have that the solution is

$$
y(x)=\sum_{n=0}^{\infty} \lambda^{n}{ }_{k} \mathbf{P}_{\rho,(v+\beta) n+\beta, \omega}^{(\delta+\gamma) n+\gamma} f(x)+c \sum_{n=0}^{\infty} \lambda^{n} x^{\frac{(v+\beta) n+\beta-k}{k}-1} E_{k, \rho,(v+\beta) n+\beta-k}^{(\delta+\gamma) n+\gamma}\left(\omega x^{\frac{\rho}{k}}\right) .
$$


To prove the theorem the following lemma is required.

Lemma 3. The Laplace transform of the $k$-Prabhakar derivative for the case $\left[\frac{\beta}{k}\right]+1=m=1$, is

$$
\mathscr{L}\left\{{ }_{k} \mathbf{D}_{\rho, \beta, \omega}^{\gamma} y(x)\right\}=(k s)^{-\frac{\beta}{k}}\left(1-\omega k(k s)^{-\frac{\rho}{k}}\right)^{\frac{\gamma}{k}} \mathscr{L}\{y(x)\}(s)-k\left({ }_{k} \mathbf{P}_{\rho, k-\beta, \omega}^{-\gamma} y\right)(0)
$$

with $\left|\omega k(k s)^{-\frac{\rho}{k}}\right|<1$.

Proof.

It is sufficient to calculate the Laplace transform of (48). To do that, we use the Laplace transform of the derivative of order $m=1$ and Lemma 2.

Proof. Applying the Laplace transform to both sides of (52)

$$
\begin{gathered}
{\left[\frac{\left.(k s)^{\frac{\beta+v}{k}}\left(1-\omega k(k s)^{-\frac{\rho}{k}}\right)^{\frac{\gamma+\delta}{k}}-\lambda\right] Y(s)=F(s)+c k,}{(k s)^{\frac{v}{k}}\left(1-\omega k(k s)^{-\frac{\rho}{k}}\right)^{\frac{\delta}{k}}}\right]\left[\frac{(k s)^{\frac{v}{k}}\left(1-\omega k(k s)^{-\frac{\rho}{k}}\right)^{\frac{\delta}{k}}}{(k s)^{\frac{\beta+v}{k}}\left(1-\omega k(k s)^{-\frac{\rho}{k}}\right)^{\frac{\gamma+\delta}{k}}-\lambda}\right] F(s),} \\
Y(s)=\left[\frac{(k s)^{\frac{v}{k}}\left(1-\omega k(k s)^{-\frac{\rho}{k}}\right)^{\frac{\delta}{k}}}{(k s)^{\frac{\beta+v}{k}}\left(1-\omega k(k s)^{-\frac{\rho}{k}}\right)^{\frac{\gamma+\delta}{k}}-\lambda}\right], \\
Y(s)=\left[\frac{(k s)^{\frac{-\beta}{k}}\left(1-\omega k(k s)^{-\frac{\rho}{k}}\right)^{-\frac{\gamma}{k}}}{1-\lambda(k s)^{-\frac{\beta+v}{k}}\left(1-\omega k(k s)^{-\frac{\rho}{k}}\right)^{-\frac{\gamma+\delta}{k}}}\right] F(s), \\
+c k\left[\frac{(k s)^{\frac{-\beta}{k}}\left(1-\omega k(k s)^{-\frac{\rho}{k}}\right)^{-\frac{\gamma}{k}}}{1-\lambda(k s)^{-\frac{\beta+v}{k}}\left(1-\omega k(k s)^{-\frac{\rho}{k}}\right)^{-\frac{\gamma+\delta}{k}}}\right] .
\end{gathered}
$$

Taking $\left|\lambda(k s)^{-\frac{\beta+v}{k}}\left(1-\omega k(k s)^{-\frac{\rho}{k}}\right)^{-\frac{\gamma+\delta}{k}}\right|<1$, we have

$$
\begin{array}{r}
Y(s)=\sum_{n=0}^{\infty} \lambda^{n}(k s)^{-\frac{(v+\beta) n+\beta}{k}}\left(1-\omega k(k s)^{-\frac{\rho}{k}}\right)^{-\frac{(\gamma+\delta) n+\gamma}{k}} F(s), \\
+c \sum_{n=0}^{\infty} \lambda^{n}(k s)^{-\frac{(v+\beta) n+\beta-k}{k}}\left(1-\omega k(k s)^{-\frac{\rho}{k}}\right)^{-\frac{(\gamma+\delta) n+\gamma}{k}} .
\end{array}
$$

Finally, by the inverse Laplace transform, we have the desired result.

Remark. We note that if $k=1, \gamma=0, \rho=\beta=1, \delta=v=2, f(x)=0, \omega=i r, \lambda=-i \pi p,(r, p \in \mathbb{R})$ (52) is

$$
\frac{d}{d x} y(x)=-i \omega \pi \int_{0}^{x}(x-t) e^{i r(x-t)} y(t) d t, y(0)=1 .
$$

which is the free electron laser equation when $x \in(0,1]$, and its solution is given in term of the k-Mittag-Leffler function

$$
E_{1,1,3 n}^{2 n}(\text { irx })
$$




\section{Conclusion}

Fractional differential operators introduced represent an interesting generalization of the Riemann-Liouville operators from the point of view of k-Fractional Calculus generated from the k-Gamma function and the Pochhammer k-symbol. These operators may be useful from a physical point of view considering the origin of these generalizations of the Gamma function and Pochhammer's symbol set by Diaz and Pariguan. Moreover, also allows us to give a new generalization of a Cauchy problem associated with the free electron laser equation.

\section{Acknowledgements}

The author wishes to acknowledge the valuable assistance of Dr. Rubén Cerutti regarding the writing of the manuscript and to the Dr. Federico Polito of the Universit degli Studi di Torino for his willingness to answer some questions, which helped to give a fair presentation of certain results.

\section{References}

[1] H. M. Srivastava and R. K. Saxena, Operators of fractional integration and their applications, App. Math. Comp. 118, 1 - 52 (2001).

[2] T. R. Prabhakar, A singular integral equation with a generalized Mittag-Leffler function in the kernel, Yokohama Math. J., 19, 171-183(1971).

[3] A. Kilbas, M. Saigo and R. K. Saxena, Generalized Mittag-Leffler function and generalized fractional calculus operators, Int. Transf. Spec. Funct. 15, 31 - 49 (2004).

[4] R. Garra, R. Gorenflo, F. Polito and Z. Tomovski, Hilfer-Prabhakar derivative and some applications, Appl. Math.Comput. 242, 576-589 (2014).

[5] C. M. Aslam and S. M. Zubair, Extended Gamma and digamma functions, Fract. Calcul. Appl. Anal., 4, 303-326 (2001).

[6] H. M. Srivastava, A. Cetinkaya and I. O. Kiymaz, A certain generalized Pochhammer symbol and its applications to hypergeometric functions, Appl. Math. Comput. 226, 484 - 491 (2014).

[7] R. Díaz and E. Pariguan, On hypergeometric functions and k-Pochhammer symbol, Divulg. Matem. 15 (2), (2007).

[8] G. Dorrego and R. Cerutti, The k-Mittag-Leffler function, J. Contemp. Math. Sci. 7, 705 - 716 (2012).

[9] R. Cerutti, On the k-Bessel functions, Int. Math. Forum, 7, 1851 - 185 (2012).

[10] G. Romero and R. Cerutti, Fractional calculus of a k-Wright type function, Int. J. Contemp. Math. Sci. 7 (31), 1547 - 1557 (2012).

[11] G. Romero, G. Dorrego and R. Cerutti, The k-Bessel functions of the first kind, Int. Math. Forum 7 (38), 1859 - 1864 (2012).

[12] G. Dorrego and R. Cerutti, k-fractional Hilfer derivative, Int. J. Math. Anal. 7, 543 - 550 (2013).

[13] G. Dorrego, Alternative definition for the k-Riemann-Liouville fractional derivative, Appl. Math. Sci. 9, 481 - 491 (2015).

[14] A. Kilbas, M. Saigo and R. K Saxena, Solution of Volterra integro-differential equations with generalized Mittag-Leffler function in the kernels, J. Int. Equ. Appl. 14, 377 - 396 (2002).

[15] S. Mubeen, G. M. Habibullah, k-Fractional integrals and application, Int. J. Contemp. Math. Sci. 7, 89 - 94 (2012).

[16] G. Romero, G. Dorrego and R. Cerutti, k-Weyl fractional integral, Int. J. Math. Anal. 6 (34), 1685 - 1691 (2012).

[17] G. Romero, L. Luque, G. Dorrego and R. Cerutti, On the k-Riemann-Liouville fractional derivative, Int. J. Cont. Math. Sci. 8, 41 - $51(2013)$. 